\title{
Primary thymic atypical carcinoid with rare multiple bone metastasis: A case report and literature review
}

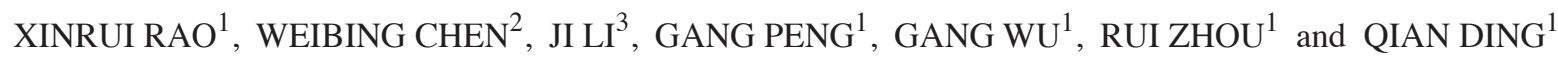 \\ ${ }^{1}$ Cancer Center, Departments of ${ }^{2}$ Pathology and ${ }^{3}$ Nuclear Medicine, Union Hospital, Tongji Medical College, \\ Huazhong University of Science and Technology, Wuhan, Hubei 430022, P.R. China
}

Received August 30, 2020; Accepted February 9, 2021

DOI: $10.3892 / \operatorname{mco} .2021 .2240$

\begin{abstract}
Primary thymic atypical carcinoid (TAC) is a type of extremely rare neuroendocrine tumor. Approximately one-third of patients with TAC tumors are asymptomatic, and no treatments of TAC have been rigorously tested in multicenter clinical trials. To date, there has been no previous case report of TACs with multiple skull metastases. The present report describes a case of a 56-year-old male patient with a primary TAC and multiple axial skeleton metastases, including multiple bilateral metastases to the parietal bones, outer wall of the orbital cavities, and the petrous apex, as well as metastases to the suprasellar region and the clivus. The histological examination confirmed the diagnosis of TAC. Single-photon emission computed tomography, computed tomography and magnetic resonance imaging scans revealed multiple bone metastases. The patient refused chemotherapy and radiotherapy and only accepted alleviative treatment, and died 89 days after diagnosis. This type of cancer is highly malignant with an extremely short natural course and high disease-related mortality. In addition, a review of the literature was performed to provide a reference for proper diagnosis and treatment of TACs.
\end{abstract}

Correspondence to: Professor Qian Ding or Dr Rui Zhou, Cancer Center, Union Hospital, Tongji Medical College, Huazhong University of Science and Technology, 1277 Jiefang Avenue, Wuhan, Hubei 430022, P.R. China

E-mail: dingqian75@163.com

E-mail: mimiruirui2@163.com

Abbreviations: TAC, thymic atypical carcinoid; NETs, neuroendocrine tumors; MEN, multiple endocrine neoplasia; CA, carbohydrate antigen; NSE, neuronspecific enolase; SPECT, single-photon emission computed tomography; CT, computed tomography; MRI, magnetic resonance image; CNI, copy number instability

Key words: thymic atypical carcinoid, multiple skull metastasis, neuroendocrine tumors, thymic tumors, thymic neuroendocrine tumors

\section{Introduction}

Carcinoids are carcinomas arising from the neuroendocrine system and are composed of cells that have features of both nerve cells and endocrine cells. Thymic carcinoids are exceedingly rare malignant tumors accounting for approximately 2 to $5 \%$ of all thymic tumors and $0.4 \%$ of all carcinoid tumors with an incidence rate of approximately 0.2 per 1,000,000 population per year (1). This type of tumor occurs over a wide age range (median age, 54 years) and has a male predilection ratio of $3: 1$ (2).

According to the WHO classification, two main carcinoid subtypes have been established: Typical and atypical. The subtypes can be distinguished based on morphology, mitosis count and the presence or absence of necrosis (3). Thymic atypical carcinoids (TACs) are considered to be well differentiated neuroendocrine carcinomas (4). The term 'atypical' is applied to those tumors observed histologically to have carcinoid architecture, increased mitotic activity, and necrosis. Compared with typical carcinoids, primary TACs are rare and more aggressive, often connected with a poorer prognosis (5); the 5-year survival rate of TAC is $\sim 80 \%$, while that of a typical carcinoid is $100 \%$ (6).

Although occurrences of TACs have been previously documented, a TAC with multiple skull metastases, including metastases to the wall of the orbital cavities, the suprasellar region, clivus, bilateral petrous apex and cervical vertebrae, has not been previously reported. This patient only survived 89 days after being diagnosed, indicating the extreme malignancy of TACs.

\section{Case report}

A 54-year-old Chinese male was admitted to Wuhan Union Hospital in November, 2019 (P.R. China) with complaints of lower back pain for over 1 year, which had worsened since the previous week, and not accompanied by cough, fever, headache, expiratory dyspnea, thoracalgia, hoarseness or dysphagia. Physical examination revealed elevated blood pressure of 146/95 mmHg. No cutaneous masses or ulcerations were identified. The range of motion activity of his waist and legs was limited because of the stiffness of his lumbar vertebrae. Beyond that, the patient felt no numbness or pain in his legs and demonstrated grade 5 muscle strength. The patient 
exhibited normal physiological reflexes, bowel movements and urine and had not lost or gained weight.

Initial laboratory findings showed elevated levels of serum carbohydrate antigen (CA) 125, CA 199, ferritin and neuron-specific enolase (NSE), which were marked as tumor biomarkers (Table I). The levels of plasma D-dimer and fibrinogen were also increased, suggesting increased blood viscosity (Table I). No blood chemistry abnormalities including $\beta$-2 microglobulin, lactate dehydrogenase and calcium concentrations were observed. In addition, the basal endocrinological examination revealed normal levels of serum cortisol, plasma adrenocorticotropic hormone $(\mathrm{ACTH})$, follicle-stimulating hormone, luteinizing hormone, thyrotropin, parathyroid hormone, renin activity, aldosterone, epinephrine, norepinephrine and dopamine. The HIV antibody tested negative.

Contrast-enhanced computed tomography (CT) of the thorax revealed that after contrast agent was administered, a mildly enhanced soft tissue mass measuring $2.1 \times 3.5 \times 3.2 \mathrm{~cm}$ with a sharp border in the left upper mediastinum next to the ascending aorta was observed, with necrosis within it. Multiple ribs and thoracic vertebrae were damaged, but no enlarged lymph nodes or lung metastases were found (Fig. 1A). Contrast-enhanced CT of the abdomen did not reveal any metastatic nodules in the liver, spleen or pancreas, nor did any enlarged lymph node in the retroperitoneum. However, multiple lumbar vertebrae damages were observed (Fig. 1B). Single-photon emission computed tomography (SPECT) revealed increased uptake of imaging agent in multiple bones, including bilateral rib bones, thoracic vertebrae, lumbar vertebrae as well as the skull, which indicated multiple bone metastases (Fig. 1C).

For diagnostic purposes, the patient underwent a CT-guided, percutaneous tissue biopsy. High-magnification optical microscopy showed a clustered growth pattern with 2 mitoses/10 high-power fields (HPFs) (Fig. 2). Immunohistochemically (Fig. 3), most tumor cells were positive for pan-cytokeratin (PCK), cytokeratin 8/18 (CK8/18), epithelial membrane antigen (EMA), cluster of differentiation 56 (CD56), and synaptophysin (Syn), and the cells had a Ki-67 labeling index (LI) of 5\%. The tissue was negative for chromogranin A, leukocyte common antigen (LCA), thyroid transcription factor-1 (TTF-1), S-100 and CD99.

Four days after the biopsy, the patient complained of a headache, which was alleviated by mannitol and dexamethasone. Contrast-enhanced magnetic resonance imaging (MRI) (Fig. 1D) scans of the head revealed bilateral damage to the parietal bones, outer wall of the orbital cavities, and petrous apex, as well as damage to the suprasellar region, clivus, and cervical vertebrae. Damages were also exhibited on multiple cranial bones (Fig. 1E). However, no meningeal or parenchymal metastases were observed.

The patient was admitted to the hospital with a pain score of 8-9. During the hospitalization, the patient was first given $200 \mathrm{mg}$ celecoxib capsules (Celebrex) orally every $12 \mathrm{~h}$, but his pain was not relived. Thus, the patient was instead given oxycodone and acetaminophen tablets (Tylenol 1\#) orally every $6 \mathrm{~h}$; however, the pain was still unbearable. Finally, the patient's pain was controlled when $30 \mathrm{mg}$ oxycodone hydrochloride prolonged-release tablets (OxyContin) were administered
Table I. Laboratory findings at admission.

\begin{tabular}{lcc}
\hline Parameters & Value & Normal range \\
\hline Serum tumor marker & & \\
AFP, $\mu \mathrm{g} / \mathrm{l}$ & 0.03 & $0.89-8.78$ \\
$\mathrm{CEA}, \mu \mathrm{g} / \mathrm{l}$ & 3.34 & $<5.0$ \\
$\mathrm{CA} 125, \mathrm{U} / \mathrm{ml}$ & $78.93^{\mathrm{a}}$ & $<35.0$ \\
$\mathrm{CA} 19-9, \mathrm{U} / \mathrm{ml}$ & $120.55^{\mathrm{a}}$ & $<37.0$ \\
$\mathrm{CA} 15-3, \mathrm{U} / \mathrm{ml}$ & 15.34 & $<31.3$ \\
FERR, $\mu \mathrm{g} / \mathrm{l}$ & $767.11^{\mathrm{a}}$ & $21.8-275$ \\
fPSA, $\mu \mathrm{g} / \mathrm{l}$ & 0.04 & $<0.93$ \\
PSA, $\mu \mathrm{g} / \mathrm{l}$ & 0.02 & $<4.00$ \\
SCC, ng/ml & 1.3 & $<1.5$ \\
CYFRA21-1, ng/ml & 1.2 & $<2.5$ \\
NSE, $\mu \mathrm{g} / \mathrm{l}$ & $34.55^{\mathrm{a}}$ & $<16.3$ \\
Blood coagulation function & & \\
TT, sec & 19.1 & $14.0-21.0$ \\
FIB, g/l & $7.14^{\mathrm{a}}$ & $2.0-4.0$ \\
APTT, sec & 36.7 & $28.0-43.5$ \\
INR & 1.07 & $0.80-1.31$ \\
PT, sec & 13.7 & $11.0-16.0$ \\
D-D, mg/l & $3.03^{\mathrm{a}}$ & $<0.5$ \\
\hline
\end{tabular}

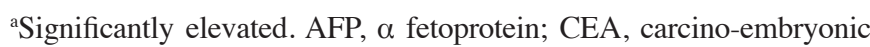
antigen; CA125, carbohydrate antigen 125; CA19-9, carbohydrate antigen 19-9; CA15-3, carbohydrate antigen 15-3; FERR, ferritin; fPSA, free prostatic-specific antigen; PSA, prostatic-specific antigen; SCC, squamous cell carcinoma antigen; CYFRA21-1, cytokerantin-19-fragment; NSE, neuronspecific enolase; TT, thrombin time; FIB, fibrinogen; APTT, activated partial thromboplastin time; INR, international normalized ratio; PT, prothrombin time; D-D, d-dimer.

orally every $12 \mathrm{~h}$ with $200 \mathrm{mg}$ Celebrex capsules, and $7.5 \mathrm{mg}$ dexamethasone pills were administered orally daily.

After the examinations were completed, the patient was diagnosed with stage IVb TAC according to Masaoka staging. Considering the lymphatic and extensive bone metastases, chemotherapy and radiotherapy were recommended to this patient. However, the patient refused to take any chemotherapy or radiotherapy and only received alleviative treatment until he died on February 20th, 2020, 89 days after he was diagnosed.

\section{Discussion}

Thymic neuroendocrine tumors (NETs) are rare tumors, In SEER database, the incidence of thymic NETs was $0.02 / 100,000$ population per year (7). Consequently, most data about thymic NETs are shown in case reports. The prevalence of thymic NETs is $\sim 3 \%$ of the total number of NETs at all sites (8). Despite the benign behavior that the name suggests, thymic NETs are known to be more aggressive than NETs in other parts of the body, frequent metastases, and occurrence as a component of multiple endocrine neoplasia-1 (MEN-1) in $\sim 25 \%$ of cases (9).

Thymic NETs are derived from Kulchitsky cells, which exist in normal thymic tissue and are part of the amine precursor 
A

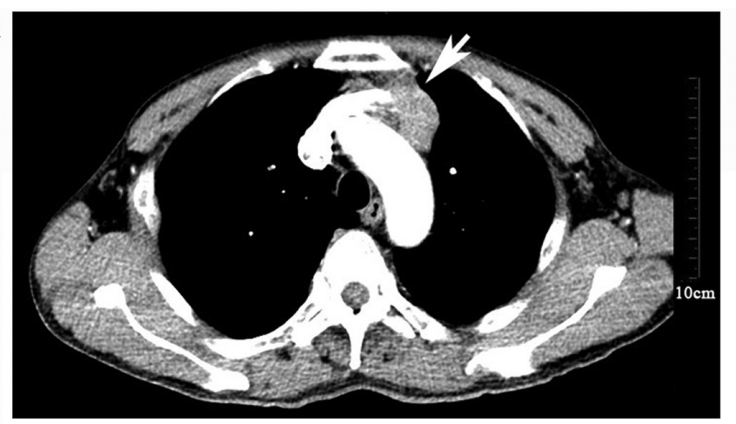

B

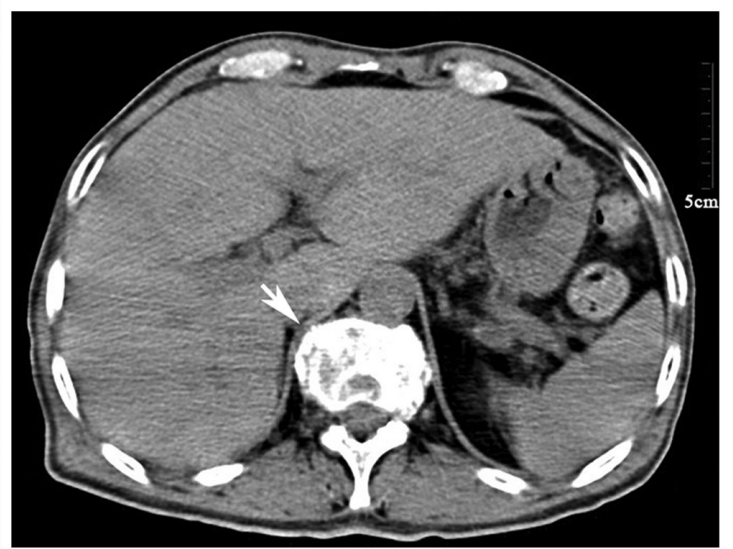

C

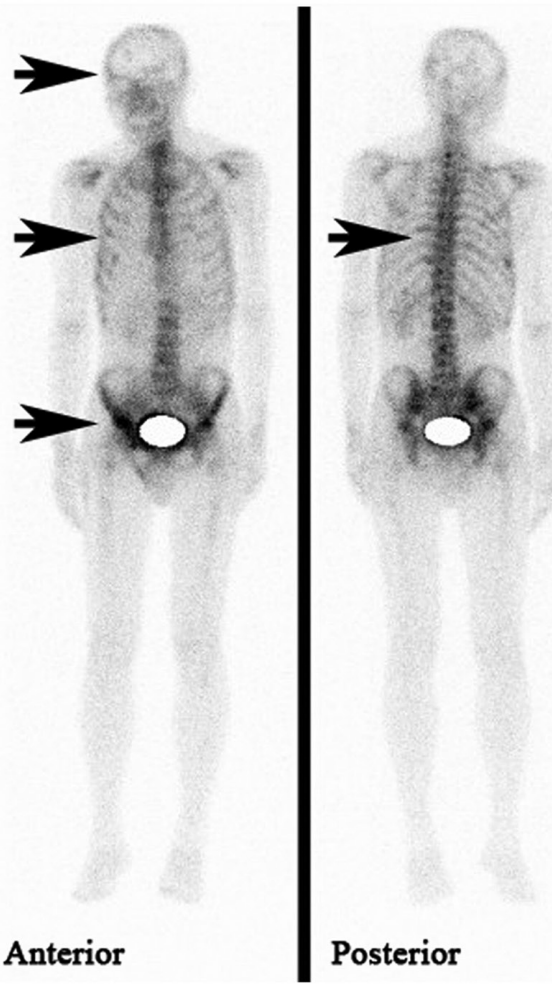

D

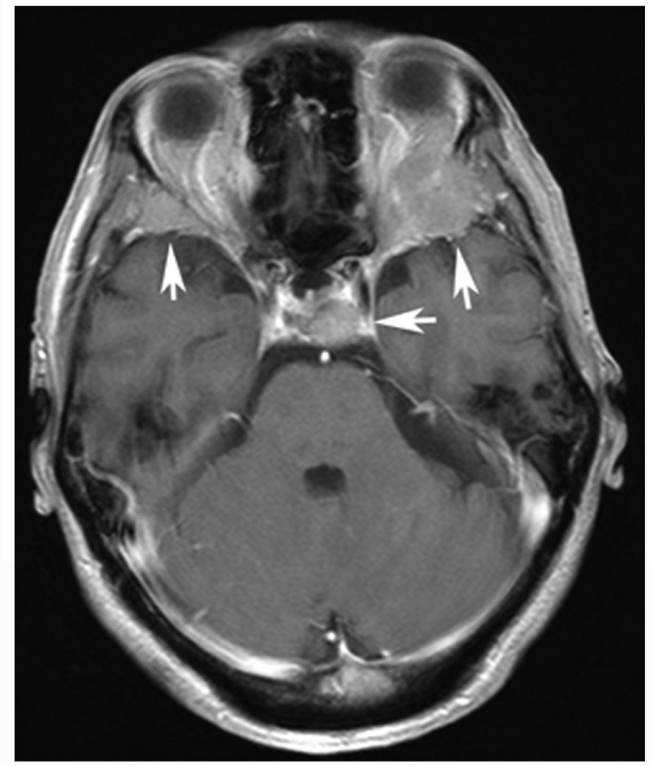

E

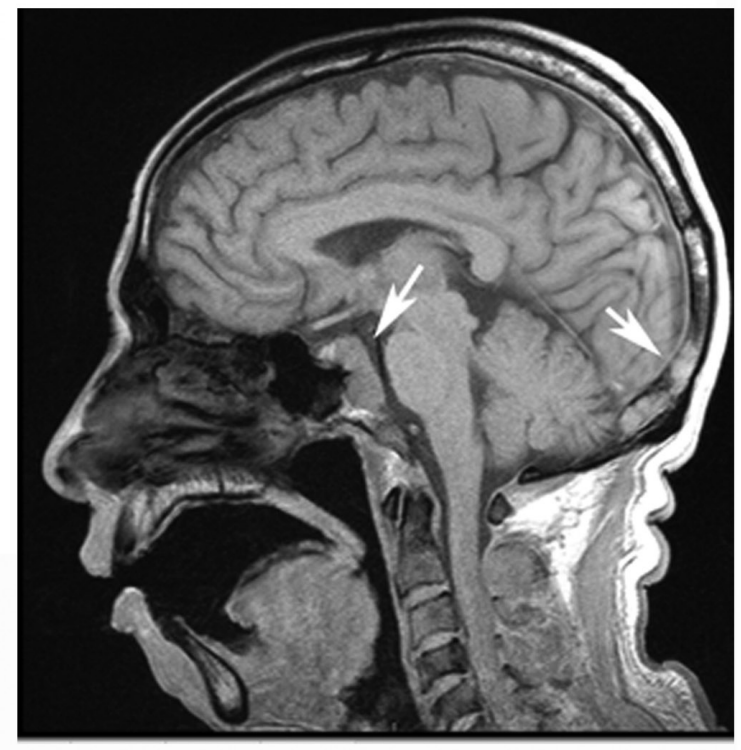

Figure 1. Typical diagnostic imaging examinations of the patient during hospitalization. (A) Contrast-enhanced thoracic CT revealing a mediastinal tumor (white arrowhead). (B) Contrast-enhanced abdominal CT revealing vertebral bone metastasis and damage (white arrowhead). (C) Bone single photon emission computed tomography revealing multiple metastases to the rib bones, thoracic vertebrae, lumbar vertebrae and skull (black arrowhead). (D) Contrast-enhanced head MRI scan revealing bilateral damage to the parietal bones, outer wall of the orbital cavities, and petrous apex, as well as damage to the suprasellar region, clivus, and cervical vertebrae (white arrowhead). (E) Contrast-enhanced head MRI scan revealing damage to multiple cranial bones (white arrowhead). CT, computed tomography; MRI, magnetic resonance imaging.

uptake and decarboxylation (APUD) system. These cells originate from neural crest cells, are readily stained with silver and are one source of foregut carcinoids (10). Thymic NETs can be categorized as typical carcinoids, atypical carcinoids, large cell neuroendocrine carcinomas, and small cell neuroendocrine carcinomas according to the WHO guidelines for bronchopulmonary NETs and thymic NETs (4), and can be further defined as well or poorly differentiated, or as type G1-4, based on the observed mitosis and Ki-67 LI. The European Society for
Medical Oncology (ESMO) clinical practice guidelines define typical carcinoids as low-grade while atypical carcinoids as intermediate-grade tumors featuring greater mitotic activity than typical carcinoids (2-9/10 HPFs vs. <2/10 HPFs), as well as focal and discrete necrosis (8). Atypical carcinoids account for $4.6 \%$ of all thymic tumors (11). Compared with lower-grade tumors, the malignant behavior of higher-grade tumors is more aggressive. Atypical carcinoid tumors of the lung and thymus show a 5-year survival rate ranging from 56 to $78 \%$, 
A

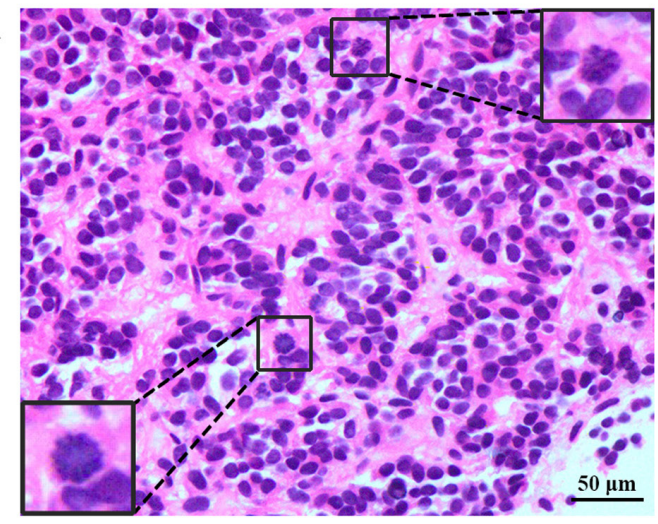

B

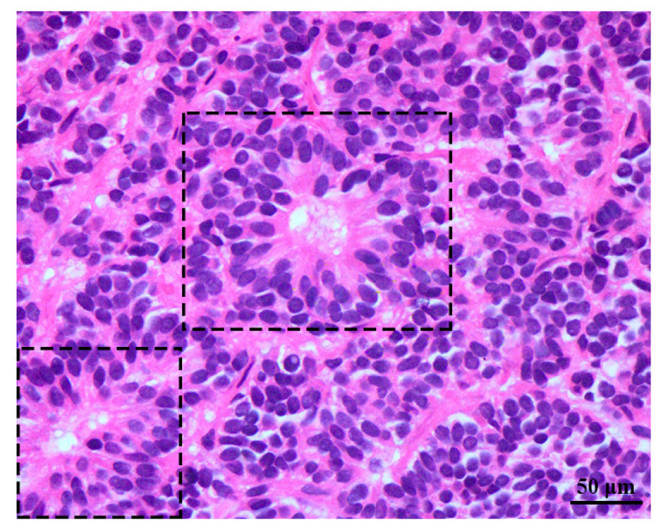

Figure 2. Histological appearance of the atypical thymic carcinoid. (A) Hematoxylin-eosin staining revealing two mitoses per high-power field (black box). (B) Hematoxylin-eosin staining revealing clustered growth pattern (black box). Magnification, $\mathrm{x} 400$.
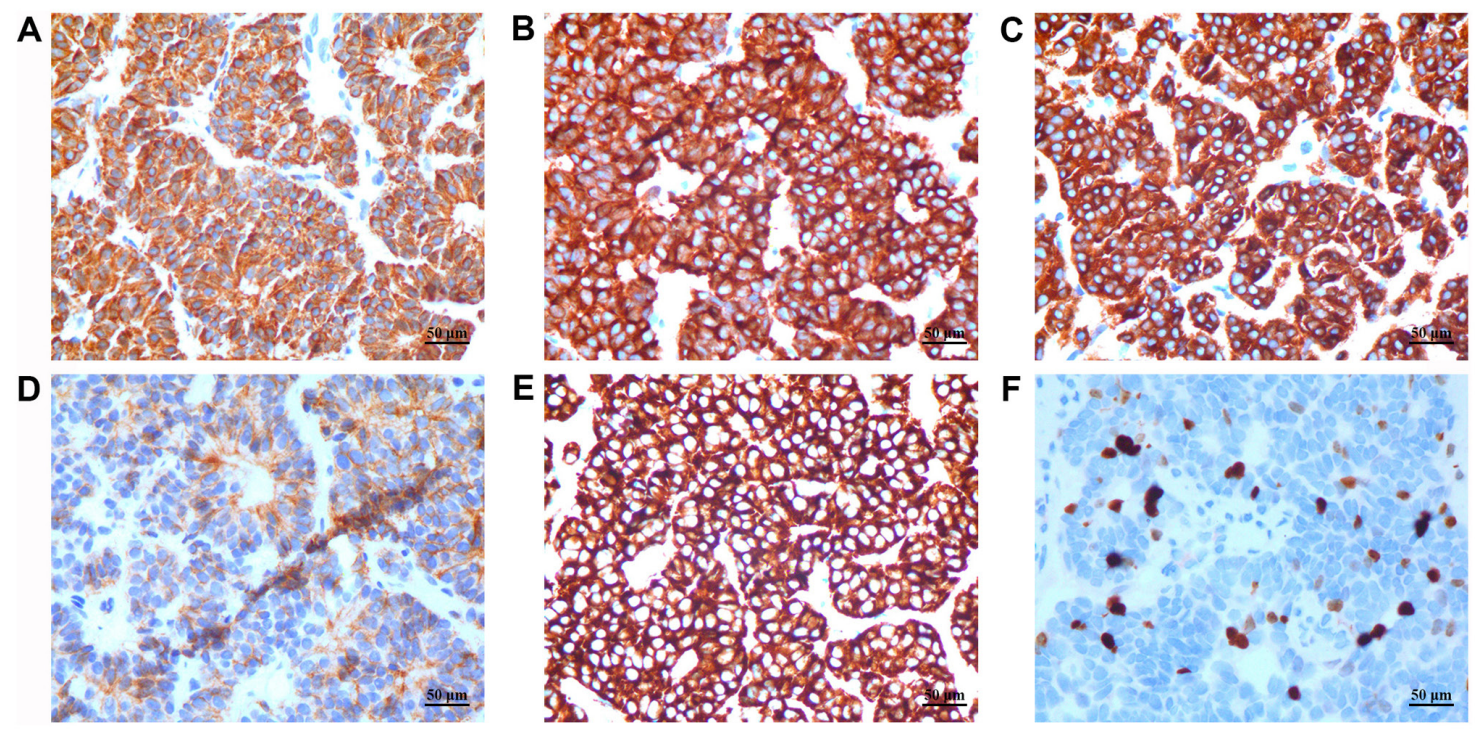

Figure 3. Immunoreactivity of the carcinoid cells for (A) pan-cytokeratin, (B) cytokeratin 8/18, (C) epithelial membrane antigen, (D) CD56, (E) Synaptophysin (Syn) and (F) Ki67. Magnification, x400.

while low-grade typical carcinoids show a $97-100 \%$ survival rate $(12,13)$.

Atypical carcinoid tumor is highly malignant and invasive without specific clinical manifestations. Since approximately one-third of patients have no obvious early symptoms and are often identified during routine health examination due to the discovery of an anterior mediastinal mass, many TAC patients already have metastases when they are diagnosed (9). Growth of the tumor mass can cause chest pain, cough or dyspnea. Patients can also be admitted with symptoms related to endocrine abnormalities (14). However, MEN-1-related TACs have been reported and are typically not accompanied by carcinoid syndromes (e.g., erythema, diarrhea, and elevated 5-hydroxyindole acetic acid), and this lack of hormonal activity probably contributes to their late diagnosis (15). In this case, this patient did not exhibit typical signs and symptoms indicating TAC because the size of the tumor in this case was relatively small and was not accompanied by endocrine abnormalities. As there were already severe damage to multiple bones at the time of the diagnosis, the patient had missed the optimal treatment stage. This case suggests the importance of routine chest X-rays or low-dose CT examinations for the early diagnosis and treatment of this disease.

Contrast-enhanced CT scanning is the initial imaging modality of choice for this disease, which can accurately show the location, size, density and shape of a tumor. This technique can also clearly illustrate the surroundings of the tumor, such as the relationship between the tumor and pericardium, great vessels and chest wall. Most thymic carcinoids are anterior mediastinal masses with an average diameter of approximately $8 \mathrm{~cm}$ (11). The tumors appear as irregular masses of uniform density in CT scans and show moderate to high, inhomogeneous enhancement when contrast agents are administered. These tumors can invade the surrounding pericardium and large vascular structures and can form mediastinal lymph node metastases $(10,16)$. With the widespread use of positron emission and computed tomography (PET/CT), the diagnosis and staging of TACs has increasingly improved. Recent studies have shown strong value of PET/CT for the assessment of intermediate- and high-grade NETs and may have prognostic 
value $(17,18)$. Because TACs exhibit high glucose uptake, metastases can be more accurately detected, including those to the lymph nodes and bones. At present, the body bone scan (as SPECT scanning) is not recommended in the guideline of thymic endocrine tumor. However, bone metastasis may be involved in the relapse and prognosis of TAC patients. A case report reported that a male TAC patient was diagnosed with bone metastasis 5 months after the second operation (19), suggesting that bone metastasis may occur at various stages and be relatively hard to discover. In our case, the patient didn't show any relative symptom indicating bone metastasis at admission, which reminds us that the full body bone scan should be considered to newly diagnosed TAC patients even without any symptom indicating bone metastasis.

CT-guided core needle biopsy is crucial because the diagnosis of TACs mainly relies on pathological examination. Upon observation, Thymic carcinoids tumor cells are round or oval and have large nuclei and finely punctate chromatin; mitosis is also visible. The cells form nests or trabecular arrangements and are distinct from fibrous tissue and blood vessels when observed using light microscopy. Immunohistochemistry is the basic tool used to diagnose carcinoids. The most commonly used markers for NETs are chromogranin, Syn, NSE and CD56. In addition, EMA, CK5/6, Ki-67, and mitotic rate are also markers frequently assessed (4).

Atypical and typical carcinoids have similar immunohistochemistry factors. However, high mitotic counts, nucleus polymorphisms, hyperpigmentation, disordered cell arrangement, focal necrosis and calcification are more common in atypical carcinoids. In our case, the immunohistochemistry results revealed that EMA, Syn, chromogranin, and CD56 were strongly positive, indicating this tumor had an endocrine phenotype, However, this patient did not present with any symptom or sign of cryptorrhea at admission, and the main characteristic of this case was severe damage to multiple bones. The relationship between the observed bone damage and the pathological characteristics remains to be explored.

Dense-core granules could be observed in the cytoplasm by electron microscopy. These neurosecretory granules participate in ectopic hormone secretion that leads to endocrine abnormalities, the most common being Cushing's syndrome (14). Although this patient described did not exhibit symptoms of endocrine abnormalities or changes in hematology upon admission, his prognosis was still extremely poor.

So far, there are few reports of genomic tests for TAC, but a recent study shows that the average copy number instability (CNI) score of TAC patients is higher than that of patients with thymic typical carcinoid, indicating that TAC has a higher degree of genomic instability. What's more, the study presented two cases whose primary and metastatic foci were classified differently according to the WHO classification, which revealed gene alterations during the metastatic process (20). In this regard, genetic factors may be involved in the process of metastasis to specific organs such as multiple bones in our case.

The diagnosis of TACs is implied by excluding other diagnoses. Thymic carcinoids need to be differentiated from numerous other types of mediastinal tumors, such as thymoma, thymic carcinoma, lymphoma, and germ cell tumors, as well as other NETs. Thymic carcinoids also need to be differentiated from other metastatic malignancies, such as small-cell lung cancer.

Complete surgical resection is strongly recommended for localized thymic carcinoids, according to the newest version of the National Comprehensive Cancer Network (NCCN) guidelines. The treatment guidelines include complete resection of the tumor and sweeping of the peripheral adipose tissue, as well as selective sweeping of the surrounding lymph nodes. For incompletely resected tumors, postoperative adjuvant chemotherapy or radiotherapy is recommended. However, no large-scale clinical trials have confirmed the effectiveness of this approach. For locoregional, unresectable disease or distant metastases, the complete surgical resection of the primary mass and metastases remains the first choice. Once surgery is no longer an option, ablative therapy is recommended. Systemic chemotherapy has been described in case reports, most frequently using temozolomide (21). At present, a phase 2 clinical trial aimed to assess the efficacy and safety of long-acting pasireotide and everolimus, given alone or in combination, in patients with advanced carcinoids of the lung or thymus is ongoing, which is the first prospective, randomized clinical trial focusing on this specific patient group (22).

Generally, the prognosis of thymic carcinoids is believed to be related to tumor volume, infiltration depth, growth site, and whether the tumor is accompanied by the carcinoid syndrome. Previous reports have indicated that a high mitotic count can be considered the most important determinant of poor prognosis for atypical carcinoids (23). But in our reported case, the mitotic rate was $2 / \mathrm{HPF}$, and Ki-67 remained at $5 \%$. These results indicated that this tumor had a relatively slow growth rate. The carcinoid was highly neuroendocrine in nature but exhibited relatively low mitosis. The malignant behavior of the TAC reported here did not match its growth speed. Thus, it is not appropriate to use the mitotic rate and $\mathrm{Ki}-67$ as indicators of malignancy on this type of tumor.

Regardless of whether endocrine abnormalities are present, once TAC patients lose the opportunity for complete tumor resection, the overall survival time dramatically decreases. Compared with patients who had resectable tumors, this patient had a very short survival time.

This patient was admitted with lumbar back pain. The contrast-enhanced chest $\mathrm{CT}$ revealed a tumor located in the left of the anterior superior septum; the longest diameter of the tumor was $3.5 \mathrm{~cm}$. There were no metastases to any lymph nodes or solid organs. CT, SPECT and MRI scan all confirmed damage to multiple bones of the spine, ribs, pelvis and skull. Bone metastasis may occur in TAC cases, but skull metastasis is rare. This is the first reported case of TAC without osteodynia as the initial symptom with damage to multiple axial bones, including metastases to the sacroiliac joint, acetabulum joint and pubis. Consequently, examination of bones is strongly recommended in this disease, even when there are no metastases to other organs.

It is the first report about primary TAC which had metastases to the parietal bones, outer wall of the orbital cavities, petrous apex, suprasellar region and the clivus. In conclusion, more clinical evidence is urgently needed to support the development of more accurate and effective means of diagnosing and treating TAC. 


\section{Acknowledgements}

Not applicable.

\section{Funding}

The present study was supported by the National Natural Science Foundation of China (grant no. 81802287; to RZ).

\section{Availability of data and materials}

The datasets used and/or analyzed during the current study are available from the corresponding author on reasonable request.

\section{Authors' contributions}

RZ and QD made substantial contributions to the conception and design of the current study. RZ curated the data. GW and GP confirmed the authenticity of all the raw data. XR, WC, GW, GP and JL performed formal analysis. XR wrote the original draft of the manuscript. RZ, GW, GP and QD critically revised the manuscript. All authors read and approved the final manuscript.

\section{Ethics approval and consent to participate}

The present study was approved by the Medical Ethics Committee of Union Hospital of Tongji Medical College, Huazhong University of Science and Technology (approval no. 2018S369; Wuhan, China).

\section{Patient consent for publication}

Written informed consent for publication of the patient's data was obtained from the patient's family member.

\section{Competing interests}

The authors declare that they have no competing interests.

\section{References}

1. Yao JC, Hassan M, Phan A, Dagohoy C, Leary C, Mares JE, Abdalla EK, Fleming JB, Vauthey JN, Rashid A and Evans DB: One hundred years after 'carcinoid': Epidemiology of and prognostic factors for neuroendocrine tumors in 35,825 cases in the United States. J Clin Oncol 26: 3063-3072, 2008.

2. Litvak A and Pietanza MC: Bronchial and thymic carcinoid tumors. Hematol Oncol Clin North Am 30: 83-102, 2016.

3. Chen G, Marx A, Chen WH, Yong J, Puppe B, Stroebel P and Mueller-Hermelink HK: New WHO histologic classification predicts prognosis of thymic epithelial tumors. Cancer 95: 420-429, 2002

4. Travis WD, Brambilla E, Nicholson AG, Yatabe Y, Austin JHM, Beasley MB, Chirieac LR, Dacic S, Duhig E, Flieder DB, et al: The 2015 world health organization classification of lung tumors: Impact of genetic, clinical and radiologic advances since the 2004 classification. J Thorac Oncol 10: 1243-1260, 2015.

5. Filosso PL, Yao X, Ahmad U, Zhan Y, Huang J, Ruffini E, Travis W, Lucchi M, Rimner A, Antonicelli A, et al: Outcome of primary neuroendocrine tumors of the thymus: A joint analysis of the International thymic malignancy interest group and the European society of thoracic surgeons databases. J Thorac Cardiovasc Surg 149: 103-109.e2, 2015.
6. Tiffet O, Nicholson AG, Ladas G, Sheppard MN and Goldstraw P: A clinicopathologic study of 12 neuroendocrine tumors arising in the thymus. Chest 124: 141-146, 2003.

7. Gaur P, Leary C and Yao JC: Thymic neuroendocrine tumors: A SEER database analysis of 160 patients. Ann Surg 251: 1117-1121, 2010.

8. Öberg K, Hellman P, Ferolla P and Papotti M; ESMO Guidelines Working Group: Neuroendocrine bronchial and thymic tumors: ESMO clinical practice guidelines for diagnosis, treatment and follow-up. Ann Oncol 23(suppl 7): vii120-vii123, 2012.

9. Jia R, Sulentic P, Xu JM and Grossman AB: Thymic neuroendocrine neoplasms: Biological behaviour and therapy. Neuroendocrinology 105: 105-114, 2017.

10. Moran CA and Suster S: Neuroendocrine carcinomas (carcinoid tumor) of the thymus. A clinicopathologic analysis of 80 cases. Am J Clin Pathol 114: 100-110, 2000.

11. Valli M, Fabris G, Dewar A, Chikte S, Fisher C, Corrin B and Sheppard MN: Atypical carcinoid tumour of the thymus: A study of eight cases. Histopathology 24: 371-375, 1994.

12. Garcia-Yuste M, Matilla JM, Cueto A, Paniagua JM, Ramos G Canizares MA and Muguruza I; Spanish Multi-centric Study of Neuroendocrine Tumours of the Lung for the Spanish Society of Pneumonology and Thoracic Surgery (EMETNE-SEPAR): Typical and atypical carcinoid tumours: Analysis of the experience of the Spanish multi-centric study of neuroendocrine tumours of the lung. Eur J Cardiothorac Surg 31: 192-197, 2007.

13. Mann M, Chowdhury B, Sullivan E, Nicklas R, Anthracite R and Meyer RJ: Serious asthma exacerbations in asthmatics treated with high-dose formoterol. Chest 124: 70-74, 2003.

14. Cardillo G, Rea F, Lucchi M, Paul MA, Margaritora S, Carleo F, Marulli G, Mussi A, Granone P and Graziano P: Primary neuroendocrine tumors of the thymus: A multicenter experience of 35 patients. Ann Thorac Surg 94: 241-246, 2012.

15. The BT: Thymic carcinoids in multiple endocrine neoplasia type 1. J Intern Med 243: 501-504, 1998.

16. Araki T, Sholl LM, Hatabu H and Nishino M: Radiological features and metastatic patterns of thymic neuroendocrine tumours. Clin Radiol 73: 479-484, 2018.

17. Sanli Y, Garg I, Kandathil A, Kendi T, Zanetti MJB, Kuyumcu S and Subramaniam RM: Neuroendocrine tumor diagnosis and management: (68)Ga-DOTATATE PET/CT. AJR Am J Roentgenol 211: 267-277, 2018.

18. Binderup T, Knigge U, Loft A, Federspiel B and Kjaer A: 18F-fluorodeoxyglucose positron emission tomography predicts survival of patients with neuroendocrine tumors. Clin Cancer Res 16: 978-985, 2010.

19. Zhu S, Wang ZT, Liu WZ, Zong SX and Li BS: Invasive atypical thymic carcinoid: Three case reports and literature review. Onco Targets Ther 9: 6171-6176, 2016.

20. Dinter H, Bohnenberger H, Beck J, Bornemann-Kolatzki K, Schütz E, Küffer S, Klein L, Franks TJ, Roden A, Emmert A, et al: Molecular classification of neuroendocrine tumors of the thymus. J Thorac Oncol 14: 1472-1483, 2019.

21. Hann CL and Forde PM: Lung and thymic carcinoids. Endocrinol Metab Clin North Am 47: 699-709, 2018.

22. Ferolla P, Brizzi MP, Meyer T, Mansoor W, Mazieres J, Do Cao C, Léna H, Berruti A, Damiano V, Buikhuisen W, et al: Efficacy and safety of long-acting pasireotide or everolimus alone or in combination in patients with advanced carcinoids of the lung and thymus (LUNA): An open-label, multicentre, randomised, phase 2 trial. Lancet Oncol 18: 1652-1664, 2017.

23. Han B, Sun JM, Ahn JS, Park K and Ahn MJ: Clinical outcomes of atypical carcinoid tumors of the lung and thymus: 7-year experience of a rare malignancy at single institute. Med Oncol 30: 479, 2013.

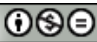

This work is licensed under a Creative Commons Attribution-NonCommercial-NoDerivatives 4.0 International (CC BY-NC-ND 4.0) License. 